\title{
CROP SPECIES IDENTIFICATION USING NN TOOL
}

\author{
Anandkumar Patil ${ }^{1}$, Lalitha Y $\mathbf{S}^{2}$ \\ ${ }^{I}$ Department of Information science and engineering, Appa institute of engineering and technology. Gulbarga. \\ Karnataka, India \\ ${ }^{2}$ Department of Electronics and Communication Engineering, Appa institute of engineering and technology. \\ Gulbarga. Karnataka, India
}

\begin{abstract}
The automatic identification of crops species is a great challenge because their patterns are complex and uncertain. In this paper the neural network is applied to identify crop species. The colour based features extraction method is a widely used for segmentation of crop images. But its performance is strongly dependent on the applied ANN technique. On the other hand, GLCM has been widely used for feature extraction because it has good performance in a large class of images. However it is not good for noisy images. In this work, colour and texture based feature algorithm for the segmentation of crop images have been proposed. The proposed method gives better results compared with techniques based on colour segmentation methods. A membership function was established. The experiment has shown that the average rate of correct identification is about to $82 \%$.
\end{abstract}

Keywords: Crops, ANN, GLCM, feature extraction.

\section{INTRODUCTION}

Farmers are the backbone of India. Crops are backbone of farmers. Using technology in the agriculture field will improve crop growth and field's fertility. Using digital image processing technology in precision farm fields, to identify crops.

In this world, we find lots of crops. However, we rarely know their names. We may consult their names with the book about crop but even such book is on hand, it is not easy to find a section or an exact page showing the crop [2][3]. It would be useful if we take a picture of the crop and feed the image into computer and computer can aid recognition and classification of the crop. In addition, some knowledge about the crop can be obtained to facilitate learning. In this study, we try to analyse various texture and colour features so that some features can be extracted, which in turn are used to achieve the goal of automatic classification of crop.

\subsection{Proposed System}

In this paper, it is proposed to find out several feature based and others are template based. For species classification, neural network based, rule based and template based methods are employed.

\section{LITERATURE SURVEY}

Many researchers working in the agriculture field. Various works Such as disease detection, weed identification etc. have been carried out in agriculture field and various image processing and machine learning techniques are being employed for this purpose. S. S. Sannakki [1] presented a study of Back Propagation Neural Network (BPNN) classifier for detection of plant diseases based on visual symptoms occurring on leaves. Images of healthy and unhealthy leaf samples are captured by digital camera, enhanced and segmented to detect infected portions. Colour and texture features are extracted and passed through BPNN classifier which correctly classifies the disease being occurred, thereby helping farmers in effective decision making. Analysis results show that the proposed classifier yields an accuracy of $97.30 \%$. Needa Samreen I.Kha [4] discussed the leaf recognition which enables the user to recognize the type of leaf using an approach that depends on neural network. Scanned images are being introduced into the computer initially, image enhancement and reduction of noise modifies their quality, further followed by feature extraction. Selection of feature points from the geometric centre of the leaf image and compares them with the already trained feature points of database leaf image is the basis for leaf recognition system. Usama Mokthar [5] introduced an efficient approach to detect and identify infected tomato leaves with these two viruses. Each input image is segmented and descriptor created for each segment. Some geometric measurements are employed to identify an optimal feature subset. Support vector machine (SVM) algorithm with different kernel functions is used for classification. The datasets of a total of 200 infected tomato leaf images with TSWV and TYLCV were used for both training and testing phase. $\mathrm{N}$-fold cross-validation technique is used to evaluate the performance of the presented approach. Experimental results showed accuracy of $90 \%$ in average and $92 \%$ based on the quadratic kernel function. Rupali R. Surase [6] developed based on Support Vector Machine (SVM) for crop classification using LISS-III imagery dataset. Several kernel functions are employed and compared for mapping the input space with including linear, sigmoid, and polynomial and Radial Basis Function (RBF). This paper illustrated the results using dataset of Aurangabad district with classification of four types of crops including cot-ton, maize, sugarcane and Bajara. Comparative analysis clearly explored that higher overall classification accuracy $(94.82 \%)$ was observed in the kernel 
based SVM compared with that of traditional pixel-based classification $(69.64 \%)$ using maximum likelihood classifier (MLC). A scaled invariant Zernike moment based feature extractor has been used to extract the relevant information from rice grain images for the purpose of classification based on Chong- Yaw Wee", Raveendran Pammesran", Fumiaki Takedab, Takeo Tsuukac, Hiroshi Kadota", Satoshi Shimanoucha"(2007). An incremental supervised learning and multidimensional maps neural network called fizzy ARTMAP (FA) has been proposed to reduce the learning time while maintaining high accuracy. A fast computation technique that uses the higher order Zernike polynomials to derive the lower order Zernike polynomial has been proposed to improve the computation speed of Zernike moments in real time applications.

The ability to recognize defining characteristics for identification is desirable as fraudulent mislabelling of rice grain varieties is a growing problem in the present work a digital imaging approach has been devised in order to investigate different types of characteristics to identify different rice varieties. Eight different common rice varieties were used in tests for defining features. These include existing standards for grain length and aspect ratio features, but also successfully show the effectiveness of compactness as a feature by D. M. Hobson, R. M. Carter, Y. Yan [16]. A novel texture feature is also shown to be able to distinguish brown and milled rice in greyscale images. All of these techniques are employed in an inexpensive imaging system that is non-intrusive and non-destructive.

In order to reduce the quantities of herbicides applied to fields, proposed to exploit the advantages of image processing to automatically detect and localize the crops and to remove all other undesired plants growing within rows and between two crops. Sajad Kiani, Zohreh Azimifar, Saadat Kamgar [10] he is stated that wavelet transformation of digital images discriminates several spatial orientations and it is very effective in analysing the information content of images. In this wavelet Analysis is used to extract appropriate features for classification. The mask is calculated for each sub-band of wavelet transform.

\section{EXPERIMENTAL SETUP}

\subsection{System Design}

The work presented here will be given by following block diagram consists of identification of crop species based on colour and texture feature.

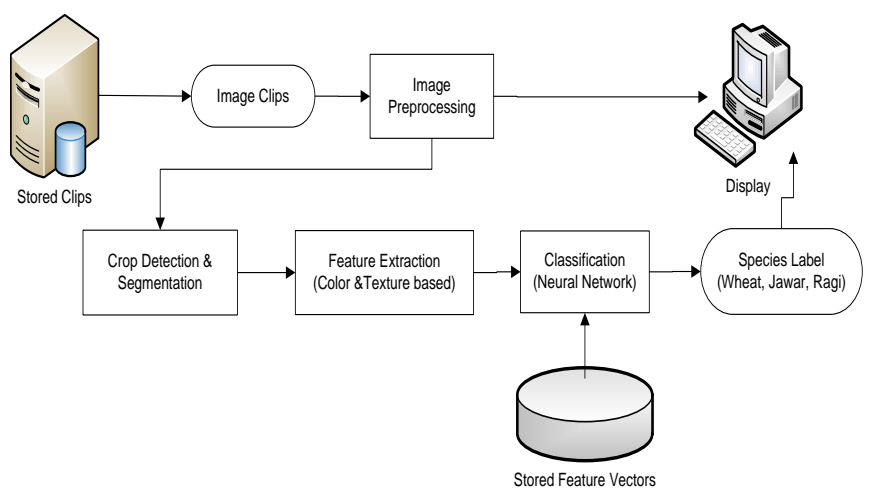

Fig 3.1.1.Block Diagram

\subsection{Image Acquisition}

For image acquisition, a colour camera was used. The images are acquired in a natural lighting condition using colour camera having resolution of 24 bits and focal length $6.3 \mathrm{~mm}$. The acquired images are of size $3264 \times 2448$ are reduced to $128 \times 128$ in order to increase the processing speed. The distance between the camera and the cops is varying. Because images are taken from angles and different distances.

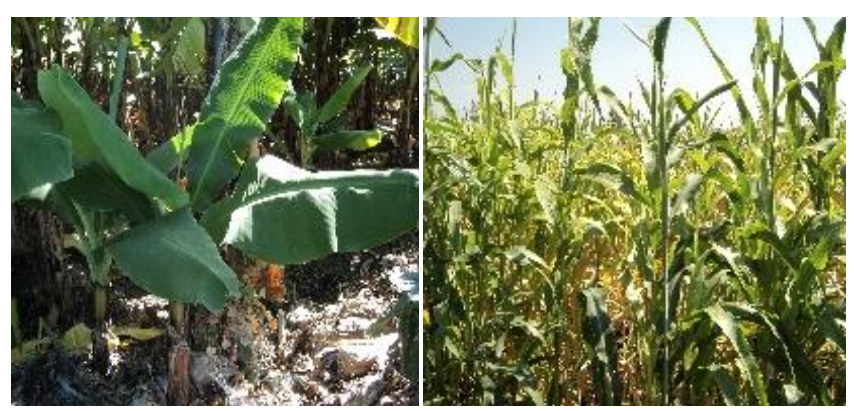

Fig 3.1.2. Crops samples.

\subsection{Data Pre-processing}

In the pre-processing stage's main purpose is to enhance the image in ways that raise the opportunity for success of the other succeeding processes (i.e. localization, segmentation, features extraction, classification, etc.).

In image processing includes,

- $\quad$ Determining ROI (Region of Interest)

- Conversion to gay scale to limit the computational requirements.

- Histogram stretching, so that the image uses the entire gray-scale. This step may not be necessary, but it is included to counter compensate for automatic light adjustment used in the used camera.

- Segmentation will divide the image into objects and regions.

\subsection{Feature Extraction}

The original 24 bit colour images are of size $M^{*} N^{*} 3$ where $\mathrm{M}$ and $\mathrm{N}$ are height and width of the image respectively and 3 indicates the three (Red, Green, Blue) 8 bit colour components of the original image. From the original image 
HIS image will be separated. The components are of size $M^{*} N$. The mean, variance and range for all these 3 components are calculated and a total of 18 colour features are stored suitably for future use. Similarly using the GLCM 24 textural features are calculated for each crop image type. Hence for every crop image total 42 features will be calculated.

\subsection{Neural Network Classifier}

The neural network architecture that is most commonly used with back propagation algorithm is the multilayer feed forward network. BPNN is best suited for agricultural applications. There are following steps in the training process.

\subsubsection{Assemble the Network and Create the}

\section{Network.}

The neural network model developed to identify the crops is shown in diagram. The number of neurons in the first layer is ' $n$ ' which is equal to dimensionality of the input pattern vectors (number of input nodes equal to number of feature nodes). The network recognizes the pattern vector $\mathrm{P}$ as belonging to class $\mathrm{O}^{\mathrm{i}}$ if the $\mathrm{i}^{\text {th }}$ output of the network is high while all other outputs are low. Due to the choice of the BPNN, the input data is normalized before being presented to the ANN. The number of nodes in the hidden layer is calcukated using the formula.

$$
\mathrm{n}=\frac{i+0}{2}+\mathrm{y}^{0.5}
$$

Where $\mathrm{n}=$ number of nodes in the hidden layer

$\mathrm{i}=$ number of input features

$\mathrm{o}=$ number of output features

$\mathrm{y}=$ number of input patterns in the training set.

For classification, the output for network is represented by 2 neurons discriminating between the classes. Each neuron is associated with one class. For example, the following resultant vector means class 1

Output $=\left[\begin{array}{ll}0 & 1\end{array}\right]$

\subsubsection{Train the Network}

The developed BPNN is trained with five different artificial and natural fruits namely apple, orange, mango, banana and pomegranate. Large number of images is required to ensure proper training of the ANNs. The extracted features of the fruits are used after normalization to train the developed model. During the training procedure of the ANNs, the maximum acceptable Mean Squared Error (MSE) is empirically set at 0.00001 . Each traverse through all of the training input and target vectors is called a pass or epoch. The training process is carried out with fast backpropagation, until a 1000 epochs (cycles) o the maximum acceptable MSE is reached. From all the training images, colour and texture features are extracted and stored in a separate databases. The combined colour and texture features are also stored in a separate database. The features extracted are given to their respective models along with expected output and its actual output is checked. If the actual output is not equal to the expected output within the specified epochs or required MSE is not reached, then the training properly.

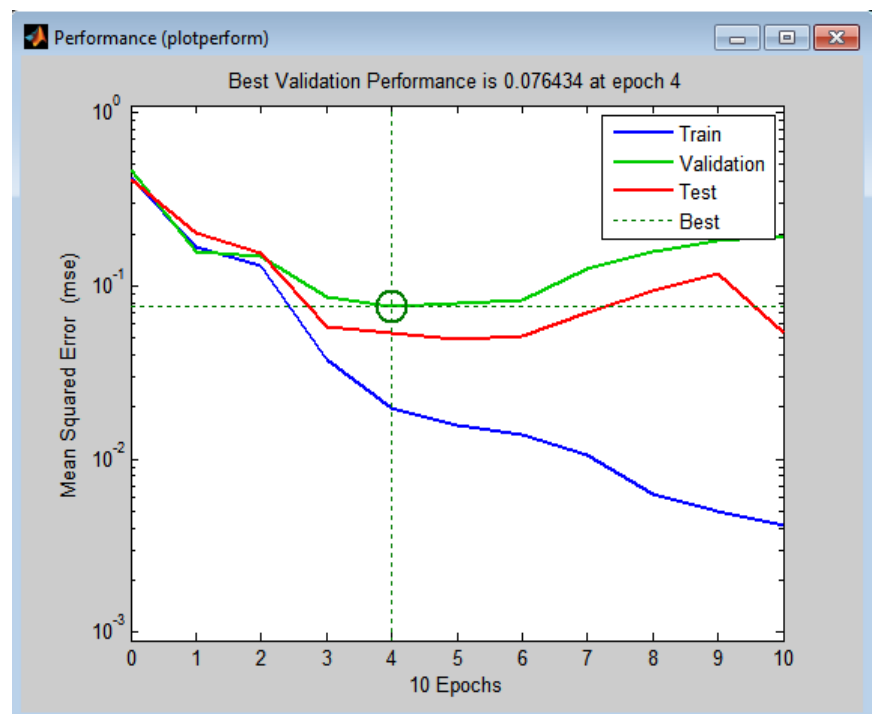

Fig 3.1.3 neural network training process

\subsubsection{Testing Phase for the BPNN}

In this phase, the crops from untrained set of samples are used to test the developed BPNN model for discrimination. From the test image, colour and texture are extracted and stored in separate files. The combined colour and texture features are also stored in separate file. The colour features are given to colour model and its output is checked. Similarly, texture, features are given to texture, combined models to combined features respectively and their outputs are tested. This process is repeated for all the testing images. While crisp values of one and zero are used in the output during training, values between zero and one can result during the testing of the ANNs. The developed BPNN model is extensively tested. During training, the ANNs are presented with binary output data.

\section{EXPERIMENTAL RESULTS}

The results obtained in this project indicates that ANN can in generally classify identification of crop species with success rates of 82 to 85 . Even though the time needed to train an ANN model is approximately 2 hours, the testing time is usually less than $1 \mathrm{~min}$ per image.

The developed neural network model is extensively trained for the set of 30 image samples and tested it for 15 image samples. The accuracy of classification is in the range of $80 \%$ to $85 \%$.

\subsection{Classification Accuracy for Crops Trained with} only Colour Features.

The graph shown in figure 4.1.1 gives the classification accuracy for crops when trained using colour features. From 
the graph, it is observed that the minimum accuracy of $50 \%$ is found with maize and maximum accuracy of $90 \%$ is found with union.

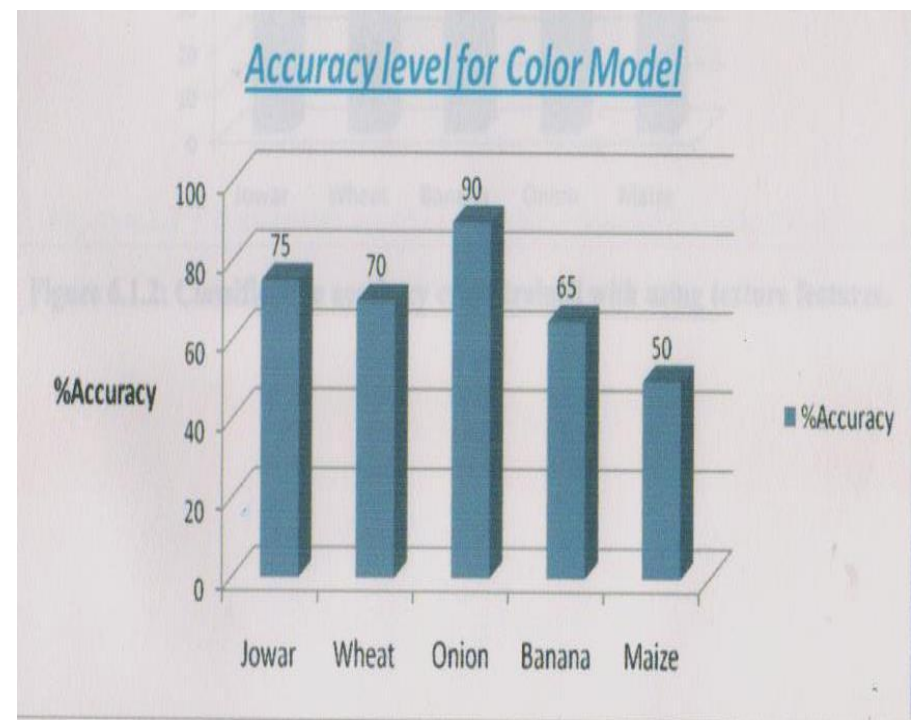

Fig 4.1.1 Ciassitication accuracy tor crops trained using colour features

\subsection{Classification Accuracy for Crops Trained with using Texture Features}

The graph shown in figure 4.2.1gives the accuracy for crops when trained with using texture features. From the graph it is observed that the minimum accuracy if $50 \%$ is found with maize and the maximum accuracy of $95 \%$ is found with onion.

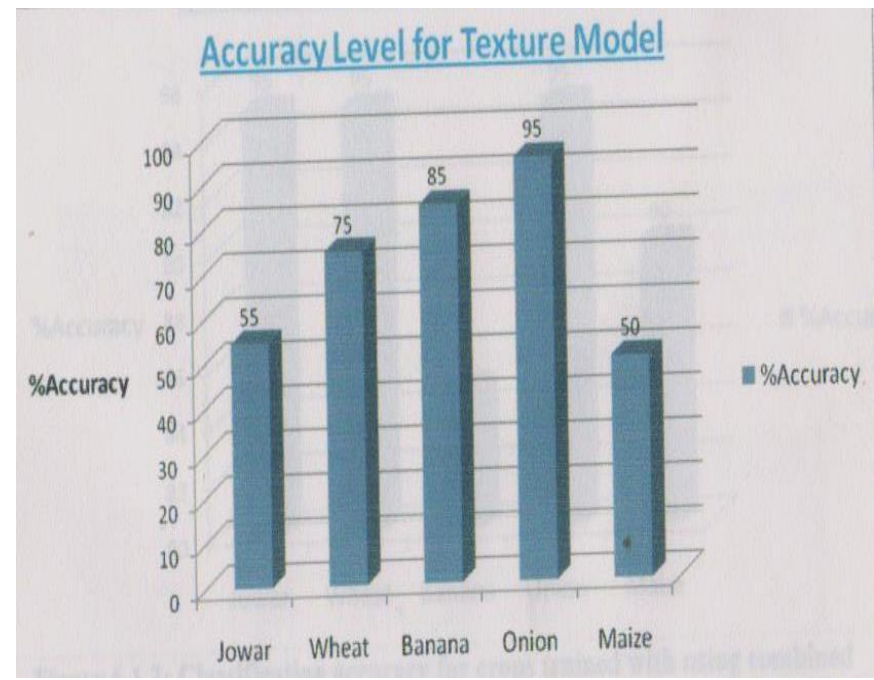

Fig 4.2.1 Classification accuracy for crops trained using texture features

\section{RESULTS SNAPSHOTS}

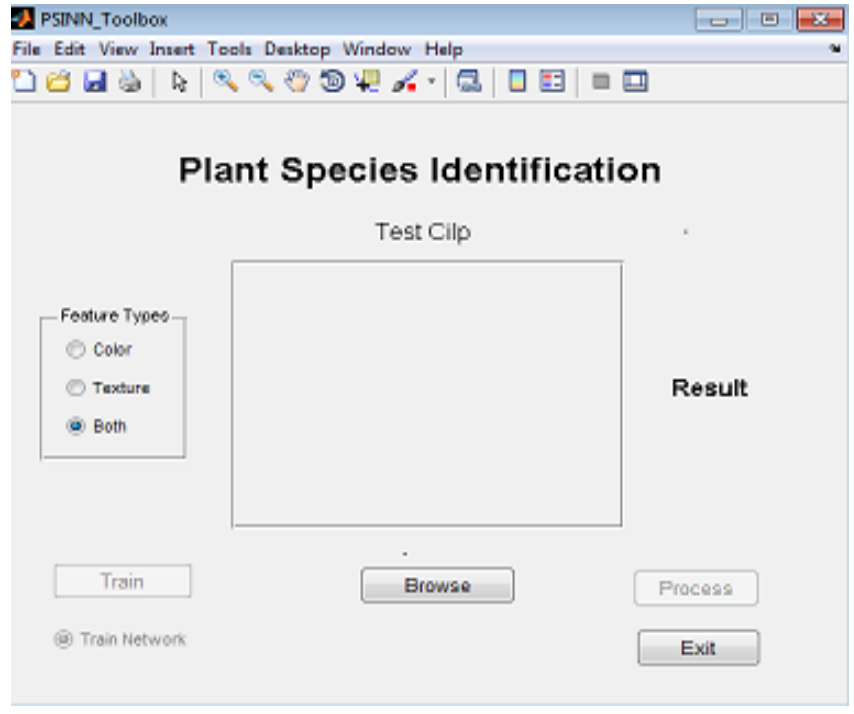

20SINN_Toolbax

File Edit View Insert Tools Desktop Window Help

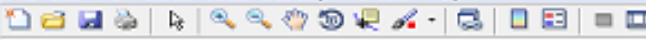

\section{Plant Species Identification}
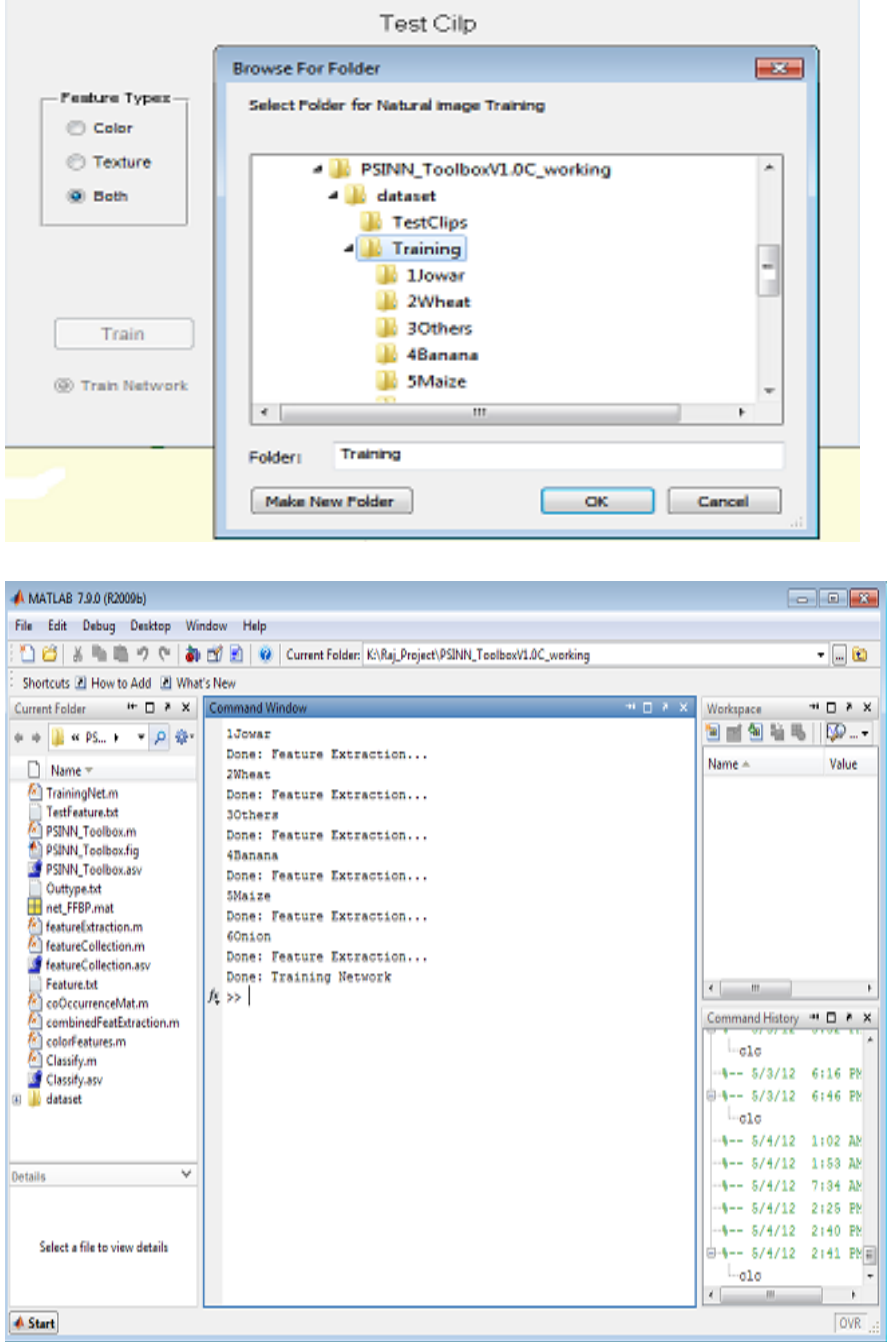

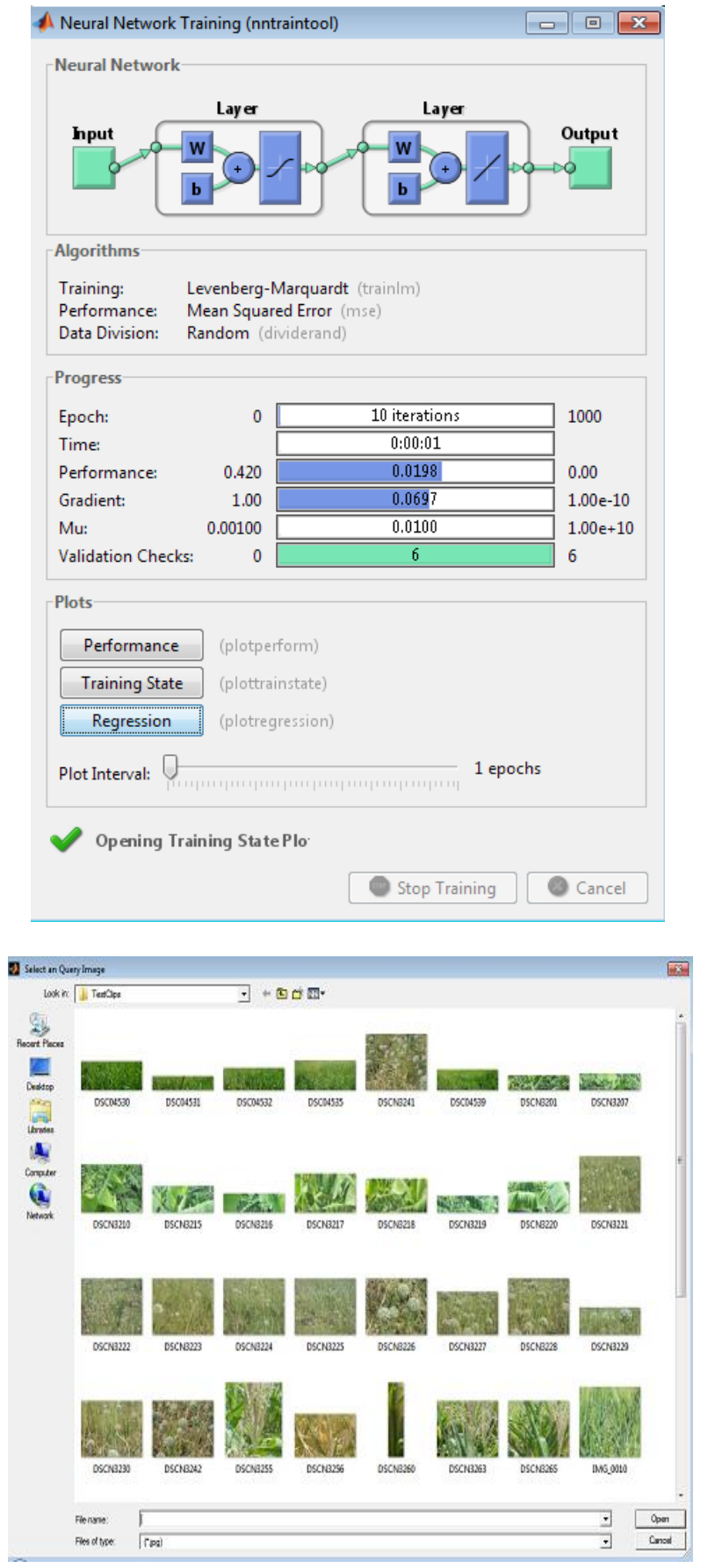

20 PSINN_Toolbox

File Edit View Insert Tools Desktop Window Help

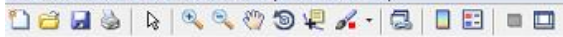

\section{Plant Species Identification}

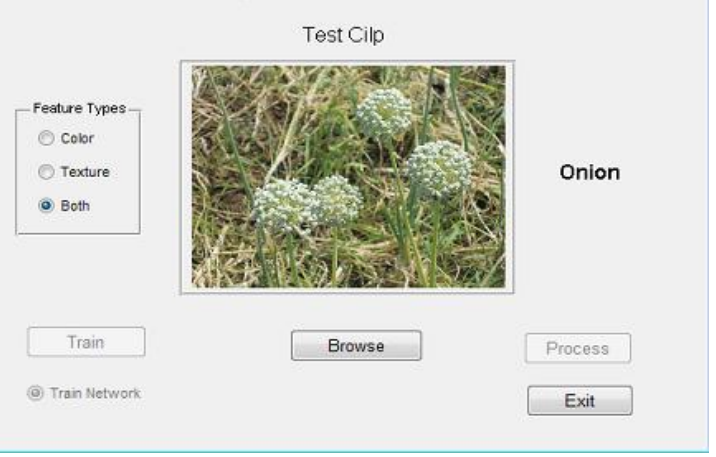

\subsection{Performance}
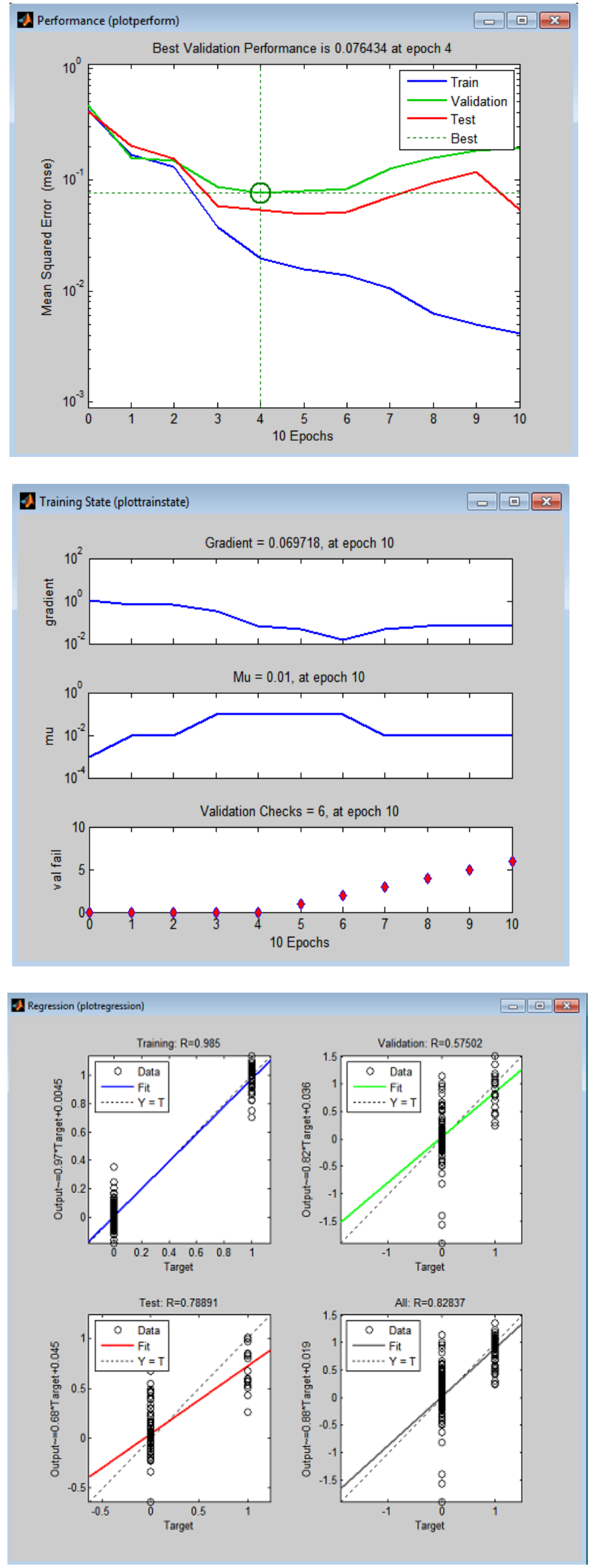


\section{CONCLUSION}

The accuracy of identification of crop species types ranging from $82 \%$ to $94 \%$. The work carried out has relevance to identification of crops and it involves both image processing and pattern recognition techniques. The images in this study are required from fruit samples. In future study can make the difference between artificial objects and natural objects. Further different natural network architecture can be used for classification. We have created 3 data sets namely colour, texture and combined feature sets. Using only colour feature set the overall accuracy for all the crop types is around $70 \%$. In this the maximum accuracy is found with onion, minimum accuracy with maize and moderate accuracies are observed for other crop types.

Using only texture feature set the overall accuracy for all the crop types is around $72 \%$. In this maximum accuracy is found with onion, minimum accuracy with maize and moderate accuracies are observed for other crop types. Using combined features set the overall accuracy for all the fruit types is around 92\%, in this maximum accuracy is found with onion, minimum accuracy with banana and moderate accuracies are observed for other crop types.

\section{FUTURE WORK}

The wok can be extended to find out many other types of crops. Similarly it can be extended to discriminate the flowers, vegetables etc. Fuzzy systems may be used and discriminate performance can be compared with neural networks.

\section{REFERENCES}

[1]. S. S. Sannakki, V. S. Rajpurohit "Classification of Pomegranate Diseases Based on Back Propagation Neural Network" IJAFRC January 2015.

[2]. SU-XIA WANG, ZHENG-HE SONG, ZHONGXIANG ZHU, BANG-JIE YANG, EN-RONG MAO, RUI ZHANG (2010).

[3]. Dr.G.Padmavathi, Mr.Muthukumar, "Image segmentation using fuzzy c means clustering method with thresholding for underwater images", Vol: 02, Issue: 02, Pages: 514-518 (2010)

[4]. Miss. Needa Samreen I.Khan, Prof. Rajesh B.Pandhare "A Review on Off-line Leaf Recognition Using Neural Network" IJCSMC, 2015

[5]. Usama Mokthar, Mona A S Ali, Aboul Ella Hassanien, Hesham Hefny "Identifying Two of Tomatoes Leaf Viruses Using Support Vector Machine”, Springer 2015

[6]. Rupali r. Surase, Karbhari v. Kale "performance evaluation of support vector machine and maximum likelihood classifier for multiple crop classification", IJRSG, 2015

[7]. M. Ameer Ali, Gour C Karmakar and Laurence S Dooley, "fuzzy image segmentation using suppressed fuzzy c-means clustering (SFCM)".

[8]. Juraj Horváth," Image Segmentation Using Fuzzy CMeans".
[9]. Jiazhi pan, Min Huang, Yong He "Crop and Weed Image Recognition by Morphological Operations and ANN model", IEEE 2007

[10]. Sajad Kiani, Zohreh Azimifar, Saadat Kamgar "Wavelet-Based Crop Detection and Classification" ICEE 2010, May 11-13, 2010

[11]. Jayamala K. Patil1 , Raj Kumar Bharti Vidyapeeth C.O.E. Kolhapur, Bhatati Vidyapeeth (Deemed Univ.) Pune Defence Institute of Advanced Tech.,Deemed University.

[12]. Javier Portilla, Vasily Strela, Martin J. Wainwright, and Eero P. Simoncelli. Image denoising using scale mixtures of gaussians in the wavelet domain.

[13]. International journal of computer applications (09758887) volume 9- no.4, november 2010 Image De-noising by Various Filters for Different Noise.

[14]. Rajesh Pydipat "Evaluation of classifiers for automatic disease detection in citrus leaves using machine vision" August 2004

[15]. Sara Verbeiren, Herman Eerens, Isabelle Piccard, Ides Bauwens , Jos Van Orshoven."Sub-pixel classification of spot-vegetation time series for the assessment of regional crop areas in Belgium", Elsevier 2006

[16]. D. M. Hobson, R. M. Carter, Y. Yan "Characterisation and Identification of Rice Grains through Digital Image Analysis", IEEE 2007.

[17]. Dengsheng Zhang,'Improving Image Retrieval Performance by Using Both Color and Texture Features" ICIG'04 IEEE 2004. 